\title{
Understanding the Systemic Effects of COVID-19: Possible Clues to Potential Therapeutic Approaches
}

\author{
Oritoke M Aluko ${ }^{1,2^{*}}$, Saheed A Lawal', Celestine S Reuben ${ }^{2}$, Sikirullai $O$ Jeje $^{1}$, and \\ Omamuyovwi $M$ ljomone ${ }^{2,3}$
}

${ }^{1}$ Department of Physiology, School of Basic Medical Sciences, College of Health Sciences, Federal University of Technology, Akure, Nigeria

${ }^{2}$ The Neuro-Lab, School of Basic Medical Sciences, College of Health Sciences, Federal University of Technology, Akure, Nigeria

${ }^{3}$ Department of Human Anatomy, School of Basic Medical Sciences, College of Health Sciences, Federal University of Technology, Akure, Nigeria

*Corresponding author: Dr. Oritoke M Aluko, Department of Physiology, School of Basic Medical Sciences, College of Health Sciences, Federal University of Technology, Akure, Nigeria, Tel: +2348067197998

\begin{abstract}
The novel coronavirus disease (COVID-19) is an infectious disease caused by the new severe acute respiratory syndrome coronavirus 2 (SARS-CoV-2). It is an acute respiratory disease that causes life-threatening symptoms. This virus directly or indirectly affects the various systems of the body. In the respiratory system, it causes pulmonary edema, impaired oxygen diffusing capacity, difficulty in breathing, etc. In the nervous system, it causes encephalitis, thrombo-embolitic stroke, Guillan-Barre syndrome (GBS), etc. In the cardiovascular system, it causes ischemic heart diseases, myocardial injury, arrhythmias, etc. In the renal system, it causes tubular necrosis, vacuole degeneration, and consequently a renal failure. In the neuromuscular system, it causes areflexia, ataxia, paresthesia, and body weaknesses. In hematological indices and immune system, it causes lymphopenia, increased monocyte and macrophages, increased neutrophils, decreased eosinophils and basophils, decreased platelet count, and exaggerated immune response. In the gastrointestinal system, it causes diarrhea, liver damage, anorexia, abdominal pain, etc. In the endocrine system, it causes acute diabetes, decreased cortisol stress response, hypothalamic-pituitary axis disorders, etc. In the reproductive system, it causes germ cell destruction, low sperm count, and erectile dysfunctions in males. In females, it causes decreased menstrual volume, cycle prolongation, preterm birth, and abnormal fetal development during pregnancy. The SARS-CoV-2 has effects on various systems of the body although the majority of the clinical manifestations of the disease are related to
\end{abstract}

the respiratory system because it is the route of entry and the major target of the disease. Meanwhile, many of its clinical symptoms are also manifested in the other systems of the body such as the nervous system, cardiovascular system, etc. Numerous researches have been done and are still ongoing on therapeutic approaches to tackle COVID-19. Here, we review the effect of COVID-19 on various physiological systems of the body and the mechanisms by which they occur. Also, we briefly highlight the potential therapeutic approaches to tackle COVID-19.

\section{Keywords}

COVID-19, SARS-CoV-2, Therapeutic approaches

\section{Introduction}

The novel coronavirus disease (COVID-19) is an infectious disease caused by the new severe acute respiratory syndrome coronavirus 2 (SARSCoV-2), which causes a severe, life-threatening acute respiratory syndrome, hyperinflammatory response, microangiopathy, damage to the blood vessels, extensive thrombosis, and angiogenesis [1]. The progression of the disease has been segmented into four stages: The first stage of upper respiratory tract infection, the second stage of dyspnea and pneumonia, the third stage characterized by worsening clinical scenario dominated by hyperinflammatory state and

Citation: Aluko OM, Lawal SA, Reuben CS, Jeje SO, ljomone OM (2022) Understanding the Systemic Effects of COVID-19: Possible Clues to Potential Therapeutic Approaches. Int J Trop Dis 5:057. doi. org/10.23937/2643-461X/1710057

Accepted: February 16, 2022; Published: February 18, 2022

Copyright: (C) 2022 Aluko OM, et al. This is an open-access article distributed under the terms of the Creative Commons Attribution License, which permits unrestricted use, distribution, and reproduction in any medium, provided the original author and source are credited. 
cytokine storm, and the fourth stage of recovery or death [1]. The outbreak of COVID-19 can be traced back to Wuhan City, Hubei Province in December 2019. The outbreak was declared by the World Health Organization (WHO) as a Public Health Emergency of International concern in January 2020 and as a Pandemic in March 2020. This disease is highly contagious and the majority of the population is susceptible to the infection. Wild animals carrying the disease and infected patients are the main sources of the disease which is transmitted through droplets from the respiratory system and direct contact with infected persons or surfaces [2,3]. General symptoms of COVID-19 include fever, cough, fatigue, difficulty in breathing, etc. However, some patients are asymptomatic or show mild symptoms, but they can spread the virus to other non-infected individuals $[4,5]$. The diagnosis of COVID-19 is confirmed using reverse transcriptase PCR, however easier methods such as rapid diagnostic test (RDT) are available [6,7]. Apart from the respiratory system, which is its main target, COVID-19 has effects on other functional systems in the body and can result in various systemic complications. Here, we review the effect of COVID-19 on various physiological systems of the body, including the respiratory, nervous, cardiovascular, renal, neuromuscular, hematology, immune, gastrointestinal, endocrine, and reproductive systems. We further review the various therapeutic approaches to tackle COVID-19.

\section{Respiratory System}

At the early stage of COVID-19 infection, SARS-CoV-2 mainly targets respiratory cells, such as pneumocytes, bronchial epithelial, and nasal cells. The structural spike protein of the virus binds to the angiotensin-converting enzyme 2 (ACE2) receptor [8,9]. The host cell's type 2 transmembrane serine protease (TMPRSS2) promotes the uptake of the virus by cleaving ACE2 and activating the SARS-CoV-2 S protein, which serves as a medium of entry for coronavirus into the host cells. Upon entry into lung tissue and cells, the viral RNA takes over the host cell's replication system to start the viral genome replication and polypeptide chain synthesis. It synthesizes the sub-

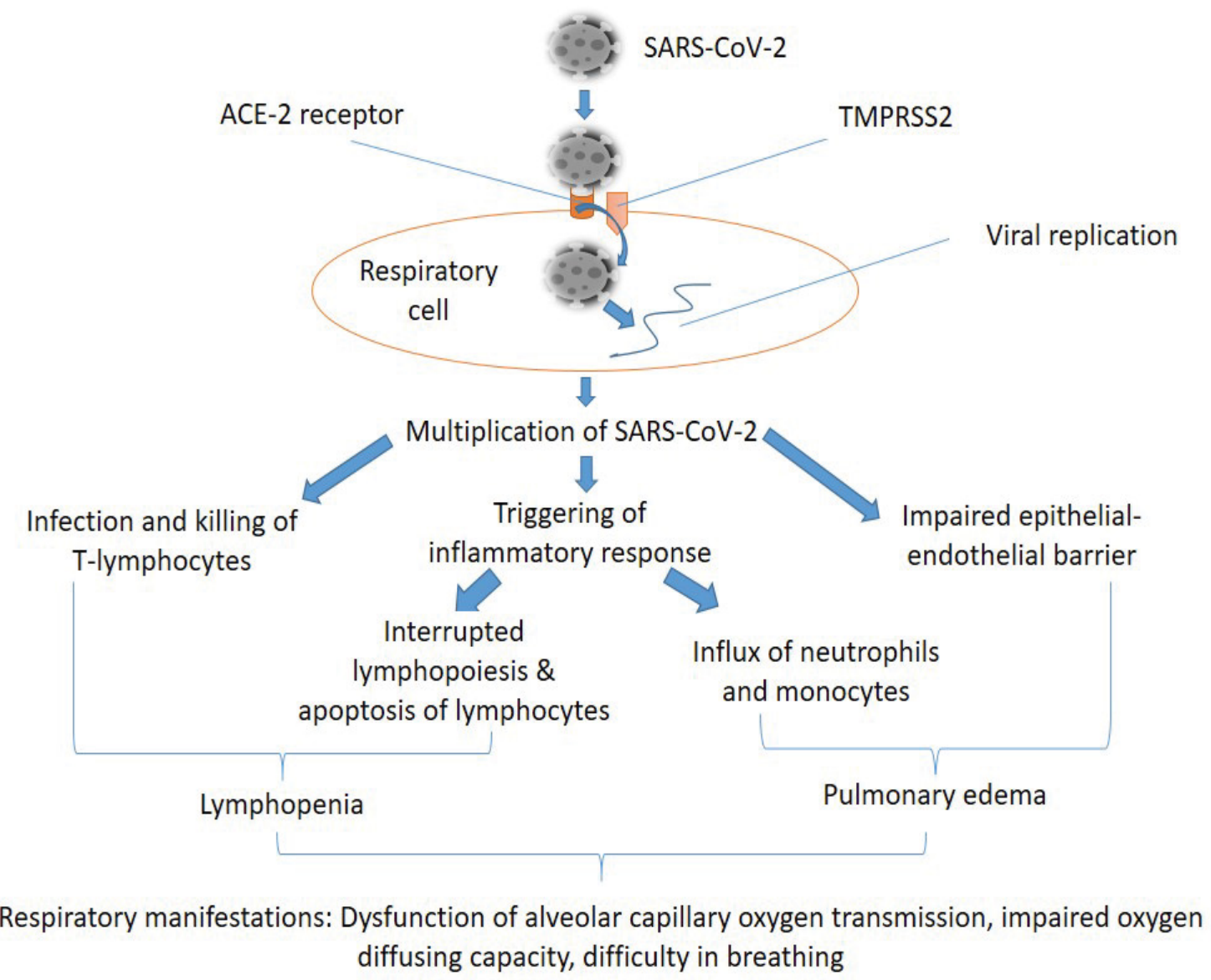

Figure 1: Mechanisms of COVID-19 effect on the respiratory system. SARS-CoV-2 enters the respiratory cells by binding to the angiotensin-converting enzyme 2 (ACE-2) receptor. The host cell's type 2 transmembrane serine protease (TMPRSS2) promotes the uptake of the virus. Upon entry into the cell, it takes over the replication system of the cell and produces its RNA and other protein materials, consequently resulting in the multiplication of the virus. The virus infects and kills T-lymphocytes, triggers an inflammatory response, and also causes impairment of the integrity of the epithelialendothelial barrier. Triggered immune response can result in interrupted lymphopoiesis and apoptosis of lymphocytes, therefore causing lymphopenia. It also results in the influx of neutrophils and monocytes, which in combination with impaired epithelial-endothelial barrier and some other processes cause pulmonary edema. A combination of the above mechanisms results in the respiratory manifestations of COVID-19, which include: Dysfunction of alveolar-capillary oxygen transmission, impaired oxygen diffusing capacity, and difficulty in breathing. 
genomic RNAs and structural proteins (envelope and nucleocapsid), which have important roles in the viral assembly, release, and promoting the pathogenesis of the virus. SARS-CoV-2 infects and kills T lymphocyte cells and also triggers an inflammatory response, including both the innate and adaptive immune response, which interrupts lymphopoiesis and accelerates apoptosis of lymphocytes, consequently resulting in lymphopenia $[9,10]$. As the infection progresses, replication of the virus increases, and the integrity of the epithelial-endothelial barrier is impaired. Pulmonary endothelial cells are also infected, thereby intensifying the inflammatory response and activating an influx of neutrophils and monocytes. Pulmonary edema develops following interstitial mononuclear cell infiltration. Edema fills the alveolar space with hyaline membrane, indicating early-phase acute respiratory distress syndrome (ARDS). Histological examination of lung biopsy from COVID-19 patients showed alveolar damage, desquamation of pneumocytes, hyaline membrane formation, pulmonary edema, and enlarged pneumocytes [11]. Figure 1 depicts the various effects of SARS-CoV-2 on the respiratory system which results in the various respiratory manifestations of COVID-19 such as disruption of the integrity of the endothelial barrier, dysfunctional alveolar-capillary oxygen transmission, impaired oxygen diffusion capacity, and difficulty in breathing [9].

\section{Cardiovascular System}

Some patients have severe damage to the cardiovascular system, and some patients with underlying cardiovascular disorders might be at risk of severe symptoms or even death. ACE2 receptor is an important respiratory receptor that is crucial in the pathogenesis of COVID-19. This receptor is not only expressed in the lungs, it is highly expressed in the heart as well. ACE2 is involved in heart function and the development of some diseases such as hypertension and diabetes mellitus. Acute myocardial injury and high blood pressure have been observed in many cases of COVID-19, especially those at the intensive care unit (ICU). The mechanism of myocardial injury has been proposed to be linked ACE2 signaling pathway, cytokine storm activated by imbalance response by type 1 and $2 \mathrm{~T}$ helper cells, and dysfunctions in the respiratory system $[12,13]$. The SARS-CoV-2 infection causes downregulation of ACE2, and it has been proposed that this down-regulation may predispose to unopposed effects of angiotensin II, such as hypertension, thrombosis, and accelerated inflammation [14]. Myocardial inflammation, metabolic dysfunction, and sympathetic nervous system activation predispose COVID-19 patients to arrhythmia. Mechanisms underlying myocardial injury are unknown or not fully understood,

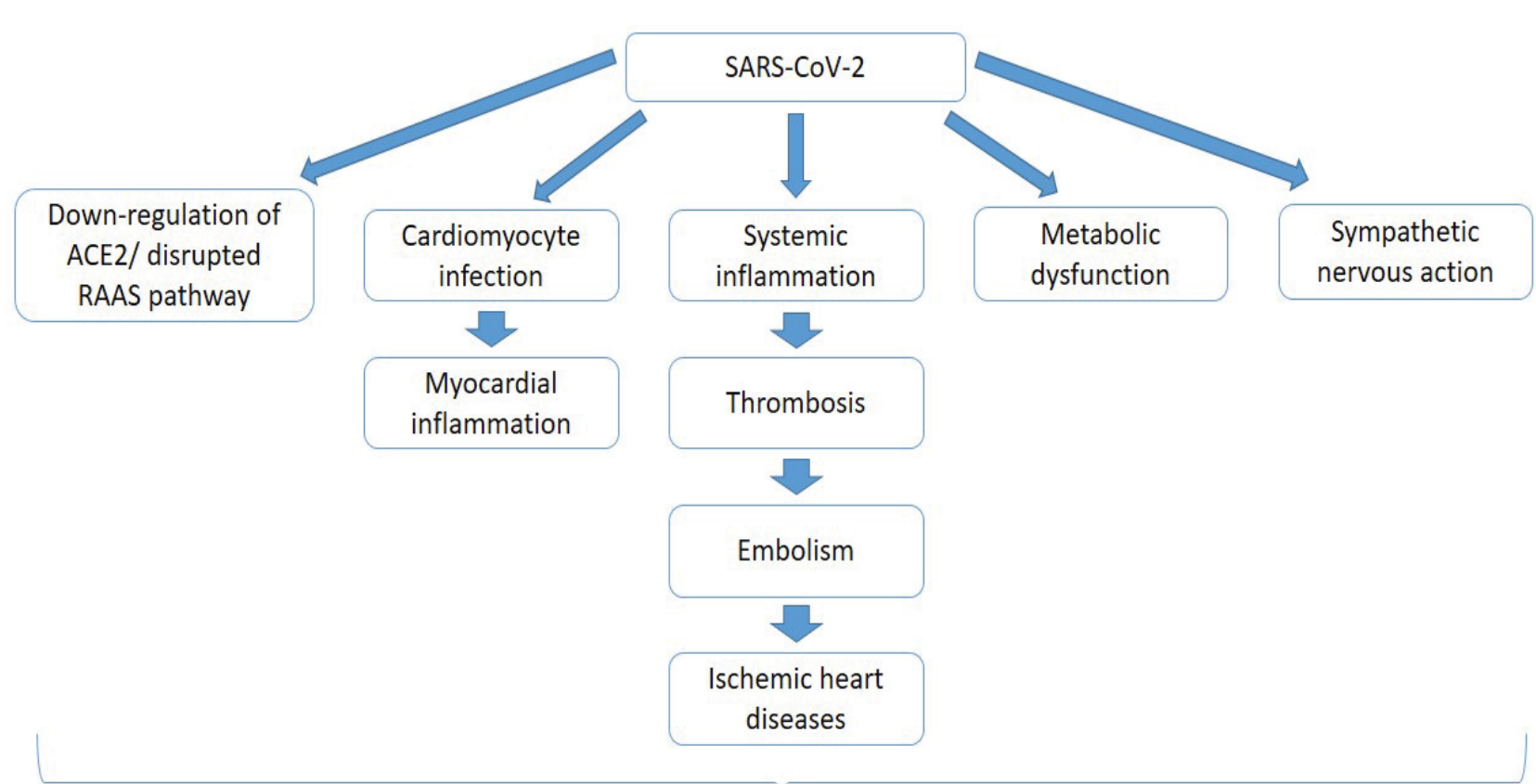

Cardiovascular manifestations: acute myocardial injury, hypertension, arrhythmias, ischemic heart diseases, etc.

Figure 2: Effect of COVID-19 on the cardiovascular system. SARS-CoV-2 affects the cardiovascular system through various mechanisms that are unknown or not fully understood. It causes down-regulation of the ACE2 receptor and imbalance in the renin-angiotensin-aldosterone system (RAAS), cardiomyocyte infection, metabolic dysfunction, and sympathetic nervous activation through cardiac autonomic functions. Cardiomyocyte infection results in myocardial inflammation. Systemic inflammation is a crucial contributor to thrombosis formation and embolism. Embolus blocks the coronary circulation and consequently results in ischemic heart diseases. All these mechanisms, though not fully understood, bring about the cardiovascular manifestations of COVID-19, which include; acute myocardial injury, hypertension, arrhythmias, ischemic heart diseases, etc. 
and a few related mechanisms are proposed. Direct cardiomyocyte infection by SARS-CoV-2 has also been considered a possible mechanism since ACE2 receptors are well expressed in the heart. SARS-CoV-2-induced systemic inflammatory response induces thrombosis formation, which is a precursor for the formation of embolism (Figure 2). Embolus plaques block the blood supply to the heart (coronary circulation), resulting in ischaemic heart disease $[9,15]$.

\section{Nervous System}

COVID-19 complications on the nervous system have emerged as an important cause of death in the pandemic as many patients exhibit neurological manifestations ranging from loss of olfactory ability and headache to disabling strokes confusion, intracranial hemorrhage, polyneuropathy, encephalitis, and GBS. The potential for central nervous system (CNS) invasion by SARS-CoV-2 has been proposed by analogy with the neurotropism of other coronaviruses, especially SARS-CoV-1, MERS-CoV, and OC43. The potential route of entry for SARS-CoV-2 includes; olfactory route, blood-brain barrier (BBB), and infiltration of infected immune cells. Loss of smell is a frequent neurological manifestation in COVID-19 and this emphasized infection of the olfactory system. Evidence of MRI signals in the olfactory cortex also suggests infections. The virus can initially be absorbed in nerve endings by endocytosis, followed by retrograde transport, and then spread trans-synaptically to other brain regions $[15,16]$. Blood-borne viruses majorly enter the brain through the BBB. Spreading of the virus into the blood has been confirmed, and the virus could access the brain by crossing the BBB. One of the potential means through which viruses enter the brain is through being carried by infected immune cells, also serving as a reservoir [15-17].

According to [18] at least four possible pathogenic mechanisms may account for the detrimental effect of COVID-19 on the CNS, which include; direct viral encephalitis, dysfunction of the peripheral organs (such as kidney, lungs, liver, etc.), systemic inflammation, and changes in the cerebrovascular structures. A single or combination of these mechanisms can put COVID-19 survivors at risk for long-term disorders of the nervous system, either by worsening a pre-existing neurological disorder or by initiating a new one. In COVID-19 patients, some cytokines and inflammatory mediators (including interleukins-1B, 2, 2 receptors, 4, 10, 18, interferon-y, etc.) are elevated, and the evidence that systemic inflammation has been proved to enhance cognitive decline and neurodegenerative disease makes it likely that survivors of COVID-19 will experience the neurodegenerative disorder in the subsequent years [18]. Various risk factors predispose COVID-19 patients to thromboembolic stroke beyond the conventional metabolic and cardiovascular co-morbidities. The thrombo-inflammatory nature of COVID-19 causes microvascular thrombosis, which in turn tends to induce systemic embolism. The formation of an embolus can interrupt cerebral circulation, consequently resulting in a stroke. The Guillian-Barre syndrome (GBS), which is made up of a group of neuropathic conditions, featured by progressive numbness and weakness, and reduced or absent myotatic reflexes, has been observed in many cases of COVID-19 [19,20]. Various studies have been made to understand the pathophysiology of GBS in COVID-19, and its mechanism has been linked to the exaggerated immune responses triggered by the virus. Critical illness polyneuropathy and myopathy (CIPNM) has also been reported in COVID-19 patients. Systemic inflammatory response mediates the release of pro-inflammatory cytokines and free radicals, which tamper with the microcirculation of the central and peripheral nervous system by diminishing nutrient and oxygen supply [20]. The underlying pathophysiology of the neurological effects of COVID-19 is yet to be fully described. Therefore, more research needs to be done to elucidate the mechanism behind the neurological manifestations. Figure 3 illustrates the pathophysiology of the effect of COVID-19 on the nervous system.

\section{Renal System}

COVID-19 has some manifestation in the renal system. Kidney damage, especially acute kidney injury (AKI) has been reported in many cases of COVID-19 [2123]. The fact that the kidney expresses the ACE2 receptor (found in vascular endothelial and smooth muscle cells, proximal epithelial cells, and podocytes) makes it susceptible to SARS-CoV-2, as this is the receptor through which the virus gains entry into the host cells. ACE2 aids the normal function of the kidney through enzymatic production of Angiotensin 1-7, which has anti-inflammatory, diuretic/natriuretic, antifibrotic and vasodilatory effects on various sections of the kidney [21-23]. Disruption of these activities makes up the proposed basis for renal damage that happens during COVID-19. The incidence of proteinuria, hematuria, elevated serum creatine, and blood urea nitrogen, and reduction in estimated glomerular filtration rate, indicate damage to the kidney in COVID-19. Postmortem autopsies of infected patients showed tubular necrosis, vacuole degeneration, sloughing of luminal brush border, and lymphocyte infiltration at the tubulointerstitium [21-23]. Direct cytotoxicity of SARS-CoV-2 or immune-mediated response is the cause of tubular damage in the kidney of COVID-19 patients $[21,24]$. It has been established that proteinuria is partly a result of direct infection of podocytes with an imbalance in the RAAS system, which together disrupts the glomerular filtration barrier and results in elevated filtration rate of plasmatic proteins. In general, the effect of COVID-19 on the renal system can be broadly attributed to three mechanisms, including; direct cytotoxic effect of the virus on the nephrons, disruption 


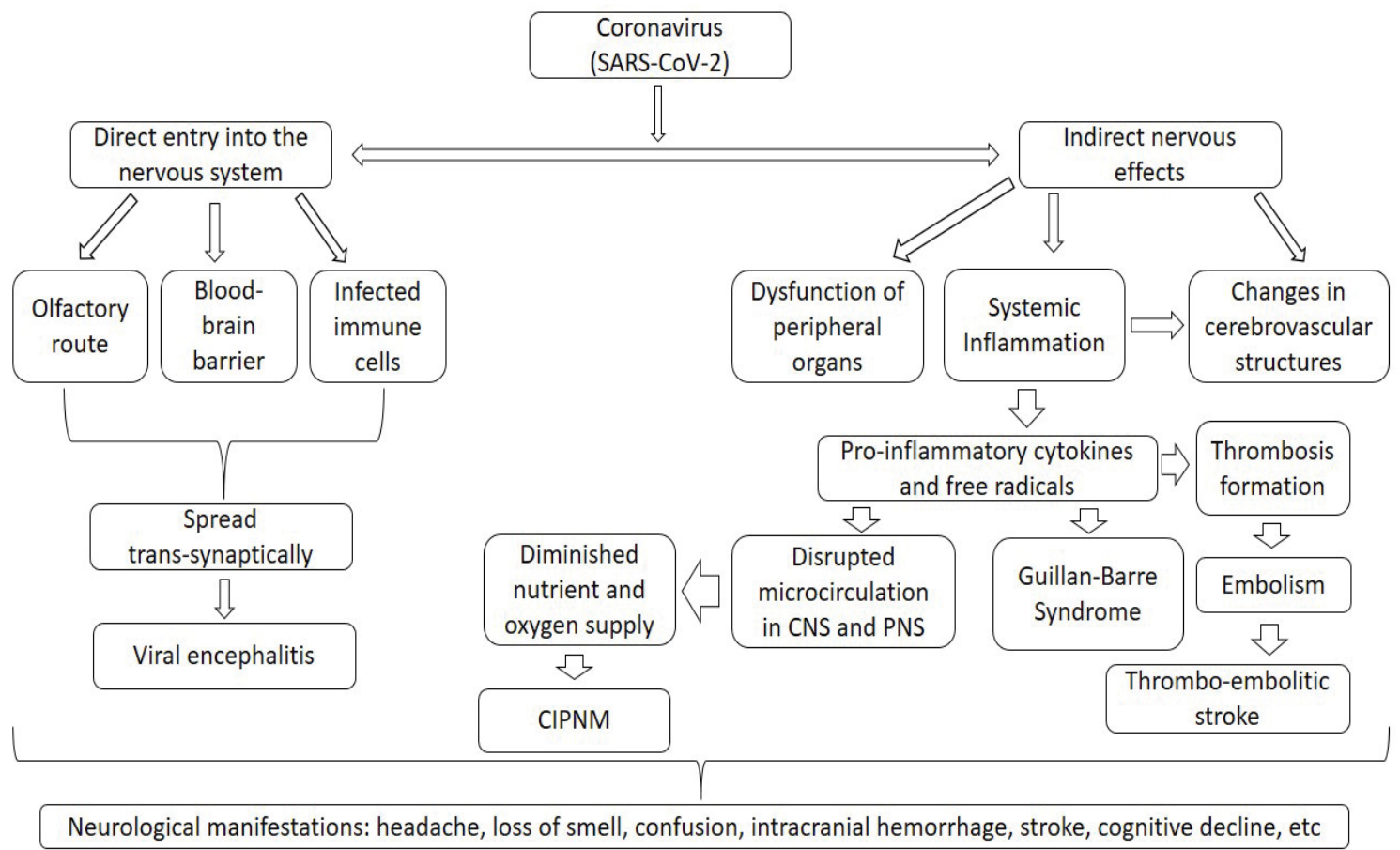

Figure 3: Mechanisms of COVID-19 effects on the nervous system. SARS-CoV-2 affects the nervous system via direct entry or indirectly through its systemic effects. Its direct entry through the olfactory system, BBB, or infected immune cells spreads trans-synaptically in the brain, infecting the neurons and consequently resulting in encephalitis. Indirectly, the SARS-CoV-2 dysfunctions in peripheral organs (such as kidney, liver, lungs, etc), have some nervous compensatory effects such as induction of systemic inflammation causing the release of pro-inflammatory cytokines and free radicals and changes in cerebrovascular structure. These pro-inflammatory cytokines can result in GBS, disrupted microcirculation in CNS, peripheral nervous system (PNS), and thrombosis formation. Thrombosis formation results in embolism and consequently thrombo-embolitic stroke. Disrupted microcirculation in CNS and PNS results in diminished nutrient and oxygen supply, consequently resulting in CIPNM. These mechanisms sum up to cause the neurological manifestation of COVID-19, which includes: Headache, loss of smell, confusion, intracranial hemorrhage, stroke, cognitive decline, etc.

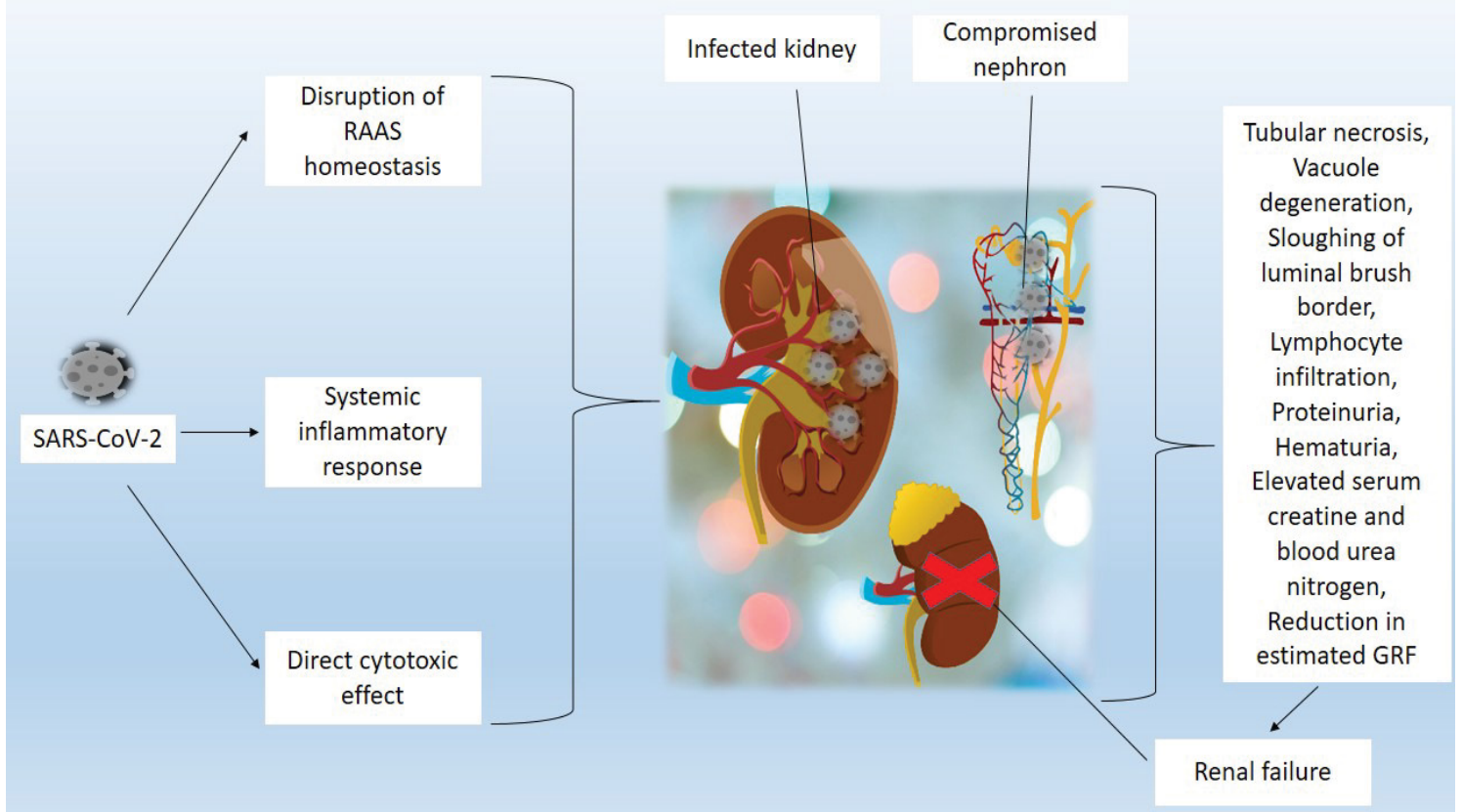

Figure 4: Mechanisms responsible for kidney damage during SARS-CoV-2 infection. SARS-CoV-2 has been described to affect the kidney through three basic mechanisms. 1) Disruption of the renin-angiotensin-aldosterone system; 2) Systemic inflammatory response, which induces cytokine storm; 3) Direct cytotoxic effect of the virus on the nephrons. These processes initiate several tissues and cellular processes that destroy the kidney by causing tubular necrosis, vacuole degeneration, sloughing of luminal brush border, lymphocyte infiltration at the tubulointerstitium, proteinuria, hematuria, elevated serum creatine and blood urea nitrogen (BUN), and reduction in estimated glomerular filtration rate. These damages consequently result in renal failure. 
of RAAS homeostasis, and systemic inflammatory response, which induces cytokine storm [25]. Figure 4 highlights the various mechanisms responsible for kidney damage during SARS-CoV-2 infection.

\section{Neuromuscular System}

Some COVID-19 patients exhibit neuromuscular disorders. These defects have been attributed to a disruption in neuromuscular transmission in the periphery as a result of peripheral neuropathy. SARS-CoV-2 triggers immune system dysregulation, cytokine storm, alteration of lymphocytes, resulting in autoimmune damage to the peripheral nervous system, and consequently neuromuscular disorders [26,27]. As discussed under the effect of COVID-19 on the nervous system, GBS is made up of a group of neuropathic conditions, featured by progressive numbness and weakness, and reduced or absent myotatic reflexes. GBS has been the major contributor to neuromuscular disorders during COVID-19. Clinical features of GBS include bilateral weakness which commences from the lower extremities and then ascends to the upper part of the body. It can also start at the proximal part of the arms or legs, and involvement of the cranial nerves can result in facial, bulbar, or oculomotor weakness. Ataxia, sensory signs, and dysfunction of autonomic control are also signs associated with GBS in COVID-19 patients $[19,28]$. Critical illness polyneuropathy and critical illness myopathy has also been observed in COVID-19 patients, especially those in the intensive care unit. According to Hasset, et al., acute inflammatory demyelinating polyneuropathy (also known as GBS), is an autoimmune demyelinating disorder of the peripheral nervous system, which is characterized by areflexia, paresthesia, and ascending body weakness that can bring about respiratory failure [29]. Figure 5 highlights the pathophysiology of neuromuscular disorders following SARS-CoV-2 infection.

\section{Hematological and Immune Systems}

Several hematological changes have been confirmed in COVID-19 patients. Most of the changes in these blood parameters occur during the progression of the disease. Many studies have shown variations in the white blood cell (WBC) count of patients. In the early stage of the disease with no symptoms, white blood cell count is normal or slightly decreased. In some studies, a higher percentage have normal ranges of WBC, patients with a decrease in WBC follow, and then the smallest percentage of the population have an increase in WBC. In other reports decreased WBC count (leukopenia) has been observed in the majority of the population, depending on the severity and progression of the disease. In some other studies, it was observed that the WBC count was higher among non-survivors of COVID-19 compared with survivors [30-32]. In COVID-19 patients, there is a decrease in the total number of lymphocytes (lymphopenia). It occurs because SARS-CoV-2 interrupts lymphopoiesis and accelerates apoptosis of lymphocytes Lymphopenia has been associated with the severity of COVID-19, and a study by [33] showed that lymphopenia increases the risk of severity of SARSCoV-2 infection threefold [31,33]. Monocyte count increases in COVID-19 patients. Activation of monocytes and macrophages in COVID-19 patients are due to acute respiratory distress syndrome and acute pulmonary

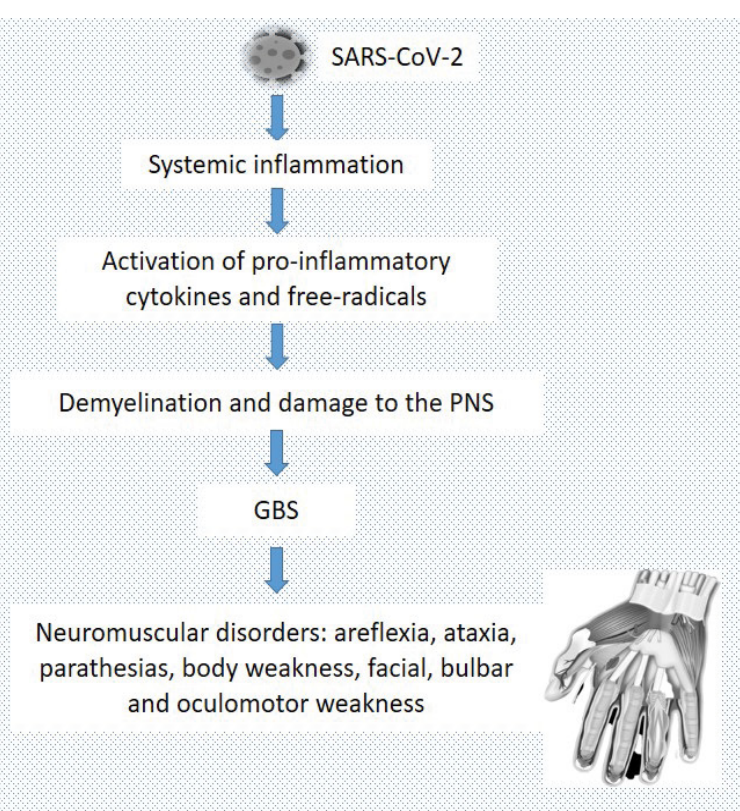

Figure 5: Pathophysiology of neuromuscular disorders following SARS-CoV-2 infection. SARS-CoV-2 triggers systemic inflammatory responses, which activate pro-inflammatory cytokines and free radicals. Pro-inflammatory cytokines and free radicals cause demyelination and damage to the PNS, resulting in GBS. 
injury. Monocytes initiate inflammation through the production of cytokines (cytokine storm) and lymphocyte activation [31,34,35]. Neutrophils are altered both numerically and morphologically in COVID-19 patients. Studies on COVID-19 patients showed an increase in neutrophil count and abnormality in their morphology. This coincides with the fact that neutrophils are always increased during infection and tissue damage. In some other studies on COVID-19 patients, eosinophil and basophil count are reduced, and it was suggested that an increase in eosinophil count could be a sign of recovery from COVID-19 [31,36]. The majority of COVID-19 studies did not consider red blood cells (RBC) as a significant parameter in the pathogenesis of the disease. Some studies mentioned that RBC count and hemoglobin level are not significantly different from the control group, while others noticed a mild decrease in RBC and hemoglobin in COVID-19 patients, which was attributed to complications from the severity of the disease. A decrease in platelet count (thrombocytopenia) is one of the clinical manifestations of COVID-19. Various mechanisms have been proposed for the decrease in platelet count in SARS-CoV-2 infection, which include; disrupted megakaryocyte maturation, accelerated platelet aggregation, platelet activation, resulting in the consumption of platelets in the microcirculation of destroyed lung tissues, inflammatory cytokines which destroy progenitor cells in the bone marrow, thereby reducing platelet production, present of antibodies and destruction of platelets [31].

The immune system plays an important role in the progression of COVID-19 and its recovery as well. Innate immune cells, such as macrophages may partake in the disease progression, while the adaptive immune responses are necessary for clearance of the SARS-CoV-2 virus. The virus triggers various innate immune recognition and response pathways. Mucosaassociated lymphoid tissues (MALT) of the oral mucosa and conjunctival epithelium serve as the first line of defense against this virus by increasing IgA response to protect these physical barriers. Infiltration of macrophages into the lung tissues is also observed in COVID-19 patients. These macrophages produce a large quantity of interleukin-6 (IL-6), which contribute to an increased inflammatory response in COVID-19. Excessive activation of inflammatory immune response leads to cytokine storm, which is has a crucial role in tissue damage and progression of the disease as stated earlier. In the adaptive immune response, it has been described that cytotoxic $\mathrm{CD}^{+} \mathrm{T}$ cells express NKG2A, TIM-3, and programmed cell death protein 1 (PD-1)

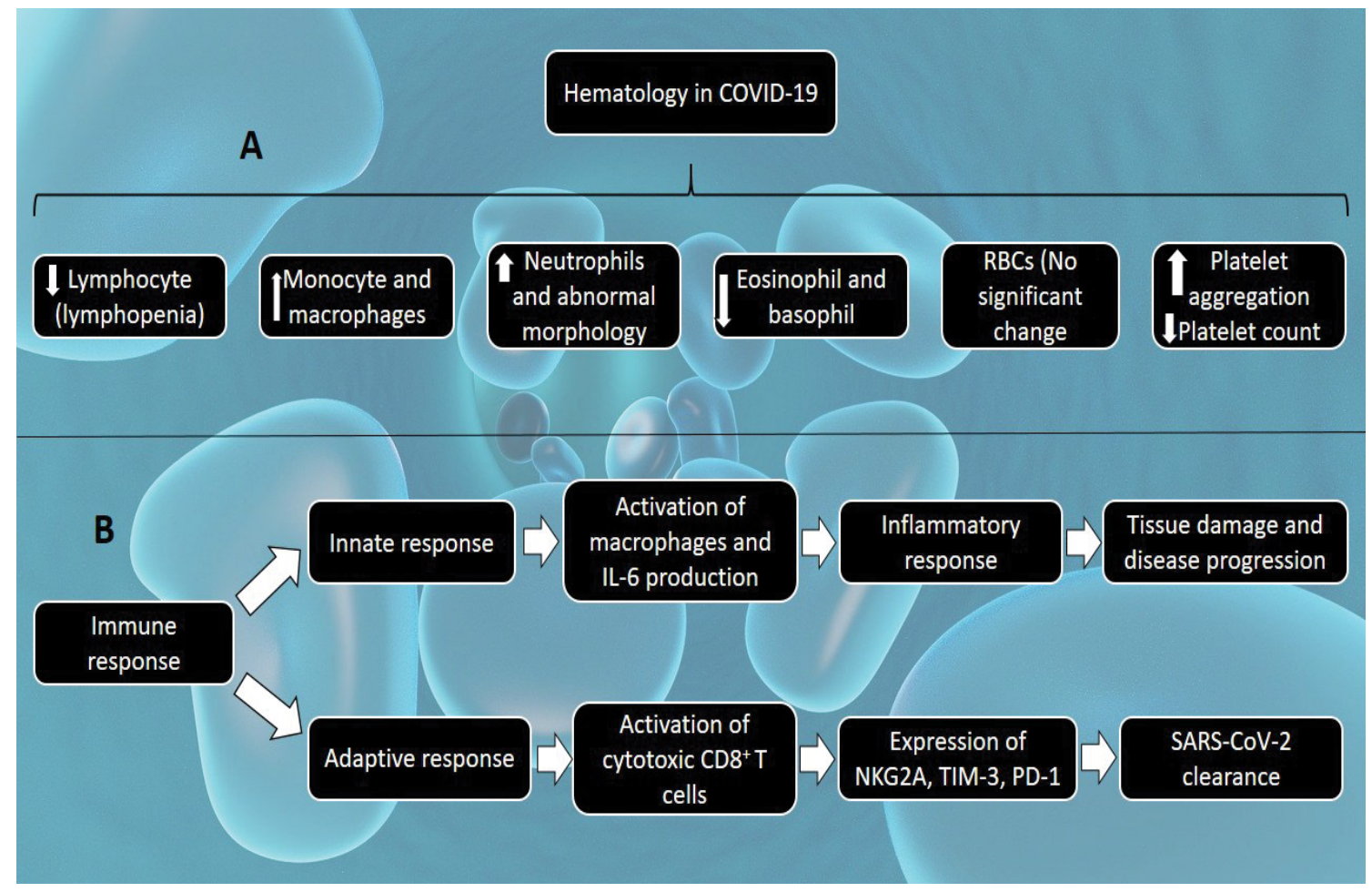

Figure 6: A) Hematological changes in COVID-19. The SARS-CoV-2 induces a decrease in the lymphocyte count, interrupts lymphopoiesis and accelerates the apoptosis of lymphocytes; an increase in monocytes and macrophages counts, as a result of acute respiratory distress syndrome and acute pulmonary injury; an increase in neutrophil count associated with abnormality in morphology; a decrease in eosinophil and basophil counts whereas an increase signifies recovery from COVID-19 infection; a decrease in platelet count; but no significant difference in the red blood cells (RBCs) count; B) Immune responses in COVID-19. Immune response in COVID-19 can be grouped into innate and adaptive immune responses. The innate response involves activation of macrophages and increased interleukin-6 (IL-6) production, resulting in the inflammatory response. This inflammatory response results in tissue damage and disease progression. The adaptive response involves activation of cytotoxic $\mathrm{CD}^{+} \mathrm{T}$ cells, which express NKG2A, TIM-3, and programmed cell death protein 1 (PD-1). These adaptive immune responses are necessary for the clearance of SARS-CoV-2. 
[37]. The immune system is highly dysregulated in severe COVID-19, contributing to the progression and pathophysiology of the disease. This pattern of the immune system is characterized by lymphopenia, IL-6mediated low HLA-DR expression, sustained cytokine production, and hyper-inflammation [35]. Figure 6A highlights the hematological changes in COVID-19 while Figure $6 \mathrm{~B}$ shows the mechanism of immune responses in COVID-19.

\section{Gastrointestinal System}

According to some reports, a little percentage of COVID-19 patients experience gastrointestinal (GI) manifestations such as diarrhea, nausea, vomiting, anorexia, and abdominal pain. The viral RNA has been found in the stool of infected patients, and liver damage has been suspected to occur. ACE 2 receptors are also expressed in the $\mathrm{Gl}$ tracts, providing evidence for a direct viral invasion of the GI tract. GI manifestations of SARS-CoV-2 may be caused either by direct invasion of the virus in the GI cells or immune-mediated tissue and organ damage [38-40]. Since some of the functions of the liver are closely associated with the gastrointestinal system, the liver is also affected by SARS-CoV-2. Injuries to the liver are not fully understood, but most of the injuries have been associated with direct viral infection of the liver cells, immune-mediated damages, or drug hepatotoxicity. Some enzymes indicating liver damage, such as alanine aminotransferase (ALT) and aspartate aminotransferase [41] are abnormal in COVID-19 patients $[40,42]$. Hepatotoxicity induced by drugs such as hydroxychloroquine and remdesivir may also contribute to elevated liver enzymes [43]. It has also been shown that COVID-19 patients with underlying chronic liver diseases may be at a higher risk of contracting the disease as a result of systemic immunodeficiency [44]. Figure 7 highlights the gastrointestinal manifestations of COVID-19.

\section{Endocrine System}

SARS-CoV-2 has some effects on the endocrine system. ACE 2 is expressed in the pancreas and the mRNA levels are higher in the pancreas than in the lungs. Damage of the pancreatic beta-cells by SARS-CoV-2 has been proposed to be responsible for acute diabetes in patients with COVID-19. Accelerated immune response during COVID-19 infection could also be responsible for the damage of the endocrine part of the pancreas. High blood glucose levels also impact the expression of ACE 2 , which might be the cause of increased incidence of COVID-19 in diabetic patients $[45,46]$. In some reports, the testosterone level was lower in COVID-19 patients, though not significant, while serum luteinizing hormone was significantly higher in COVID-19 patients compared to healthy men. COVID-19 patients with severe conditions are prone to have critical illness-related corticosteroid insufficiency ( $\mathrm{CIRCl})$ as a result of knockdown of the host's cortisol stress response. This is a major immuno-invasive technique utilized by SARS-CoV-2. A small number of COVID-19 patients have shown certain

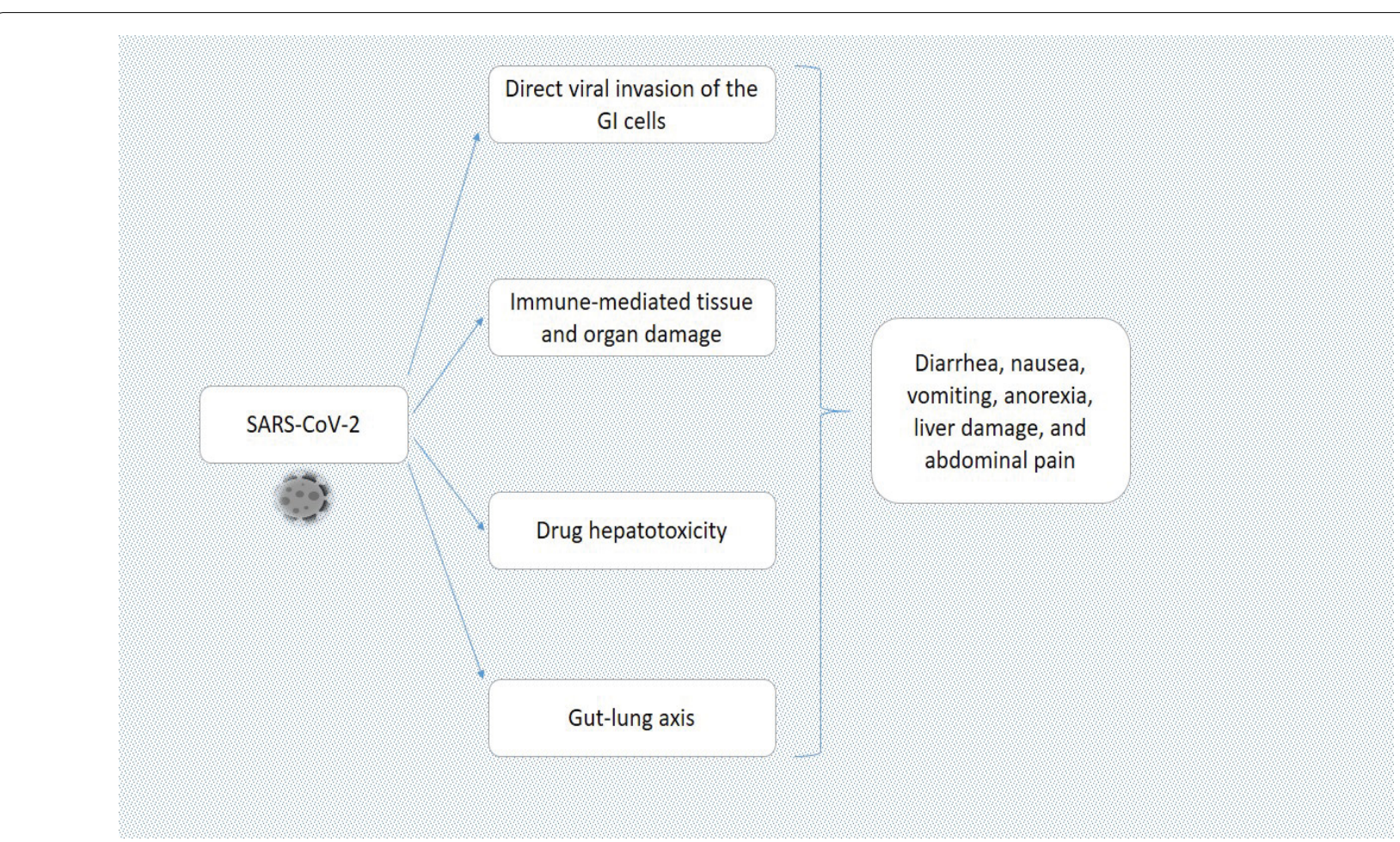

Figure 7: Mechanism of Gastrointestinal manifestations of COVID-19. SARS-CoV-2 can affect the gastrointestinal (GI) tracts through the following mechanisms; direct viral invasion of the GI cells, immune-mediated tissue and organ damage, hepatotoxicity resulting from the drugs taken during COVID-19, and the communication between the lungs and the GI tract (gut-lung axis). These mechanisms individually or together result in various GI manifestations of COVID-19, including; diarrhea, nausea, anorexia, liver damage, abdominal pain, etc. 


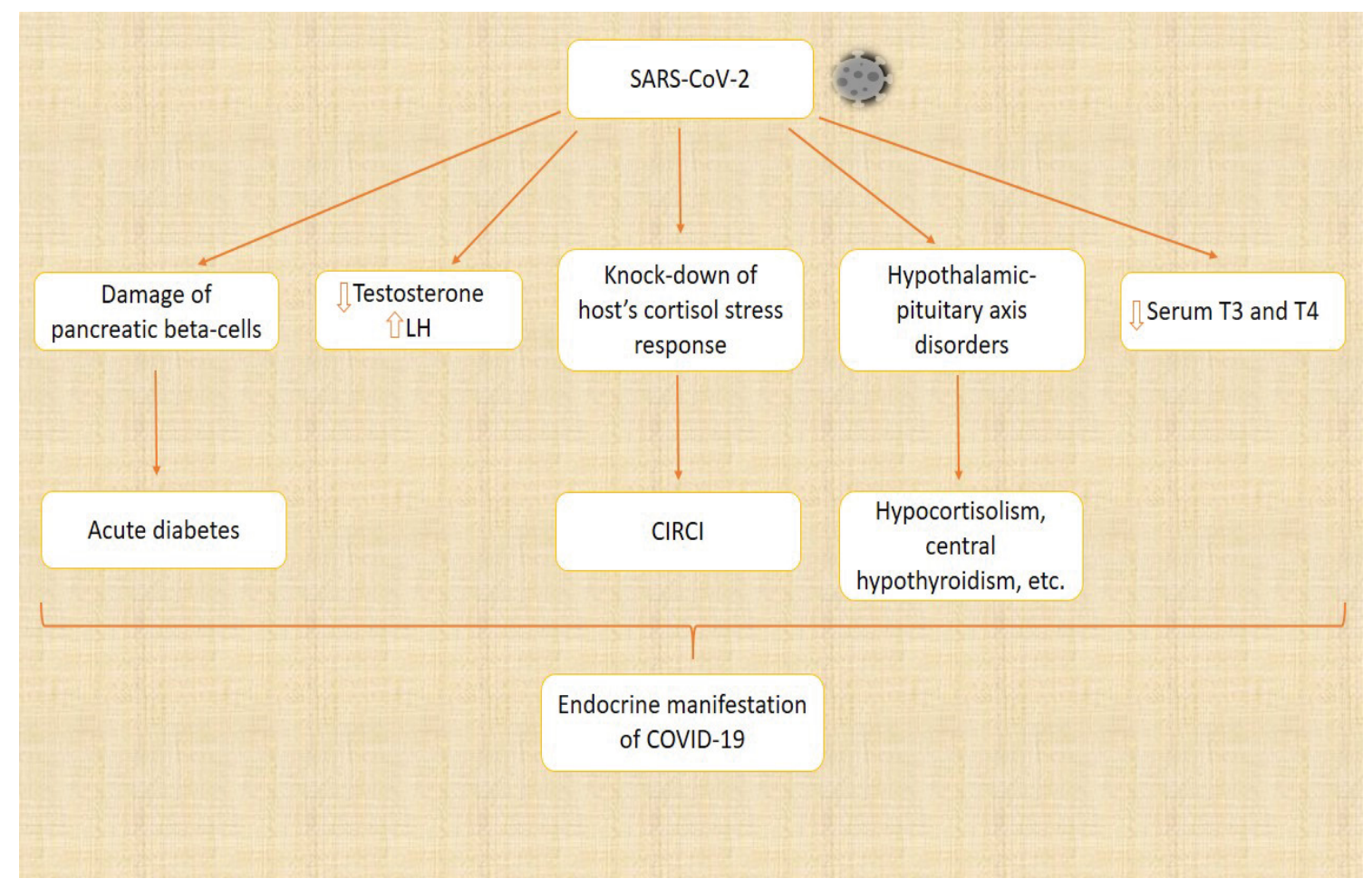

Figure 8: Endocrine changes in COVID-19. SARS-CoV-2 elicits various changes in the endocrine system, and these changes are responsible for the endocrine manifestations of COVID-19. SARS-CoV-2 destroys the endocrine pancreas, resulting in acute diabetes. The level of testosterone was also found to be lower, while the luteinizing hormone (LH) was significantly higher when compared to healthy individuals. One of the immuno-invasive methods used by SARS-CoV-2 is to knock down the host's cortisol stress response, leading to critical illness-related corticosteroid insufficiency $(\mathrm{CIRCI})$. The hypothalamic-pituitary axis is also disturbed, causing hypocortisolism, central hypothyroidism, and other disorders. The serum triiodothyronine (T3) and tetraiodothyronine (T4) were also found to be lower.

symptoms of hypothalamic-pituitary axis disorders, such as hypocortisolism, central hypothyroidism, etc. Serum triiodothyronine (T3) and tetraiodothyronine (T4) were also found to be lower in COVID-19 patients [46]. The immunosuppressive effect of androgen makes the male more susceptible to SARS-CoV-2 infection. Estrogen depletion in mice has been reported to cause morbidity and mortality, suggesting a protective impact on the estrogen receptor signaling pathway. Oxytocin has proved to be relevant in suppressing SARS-CoV-2 infection. It helps to mobilize the immune defenses and also suppresses pathogenic responses resulting from increased activation of innate immunity [47]. Melatonin is also known to protect against SARS-CoV-2 due to its anti-inflammatory and anti-oxidative effects. It also regulates neutrophils and other immune cells. Figure 8 shows the endocrine changes in COVID-19 $[47,48]$.

\section{Reproductive System}

SARS-CoV-2 has been shown to have various effects on male and female reproductive systems. ACE2 is highly expressed in the testes, especially on the surface of spermatogonia and supporting cells. This virus, as mentioned earlier can enter the body cells through the ACE2 receptor and can also trigger an immune response, leading to dysfunction in testicular spermatogenesis and low sperm count [49]. A hormonal profile has also been used to analyze the male gonadal function of COVID-19 patients. COVID-19 patients showed decreased testosterone levels and increased LH levels when compared with healthy individuals [50]. Vascular damage that occurs during COVID-19 is likely to affect the delicate vascular bed of the penis, leading to erectile dysfunction. This is because the integrity of the vascular bed is necessary for proper erectile function in men [51]. Inflammatory response by inflammatory cytokines can cause testicular damage as a result of high temperature-induced germ cell destruction, and infiltration of leukocytes, destroying the Leydig cells [52]. In the female reproductive system, ACE2 has widely expressed ovary, uterus, vagina, and even the placental during pregnancy [53]. During pregnancy, viruses have negative impacts on the fetus and the mother. An elevated level of maternal inflammation in response to SARS-CoV-2 infection can affect fetal development, especially the development of the brain, leading to several neurodevelopmental disorders in offspring. Preterm birth can also result from the complication of SARS-CoV-2 infection [41]. The report showed that pregnant women are more likely to exhibit severe COVID-19 symptoms with several pregnancy complications [54]. In a study conducted by Li, et al. on women of reproductive age that have been diagnosed with COVID-19, about $20 \%$ of them showed a decrease in menstrual volume or cycle prolongation. This was proposed to result from the changes in sex hormones 


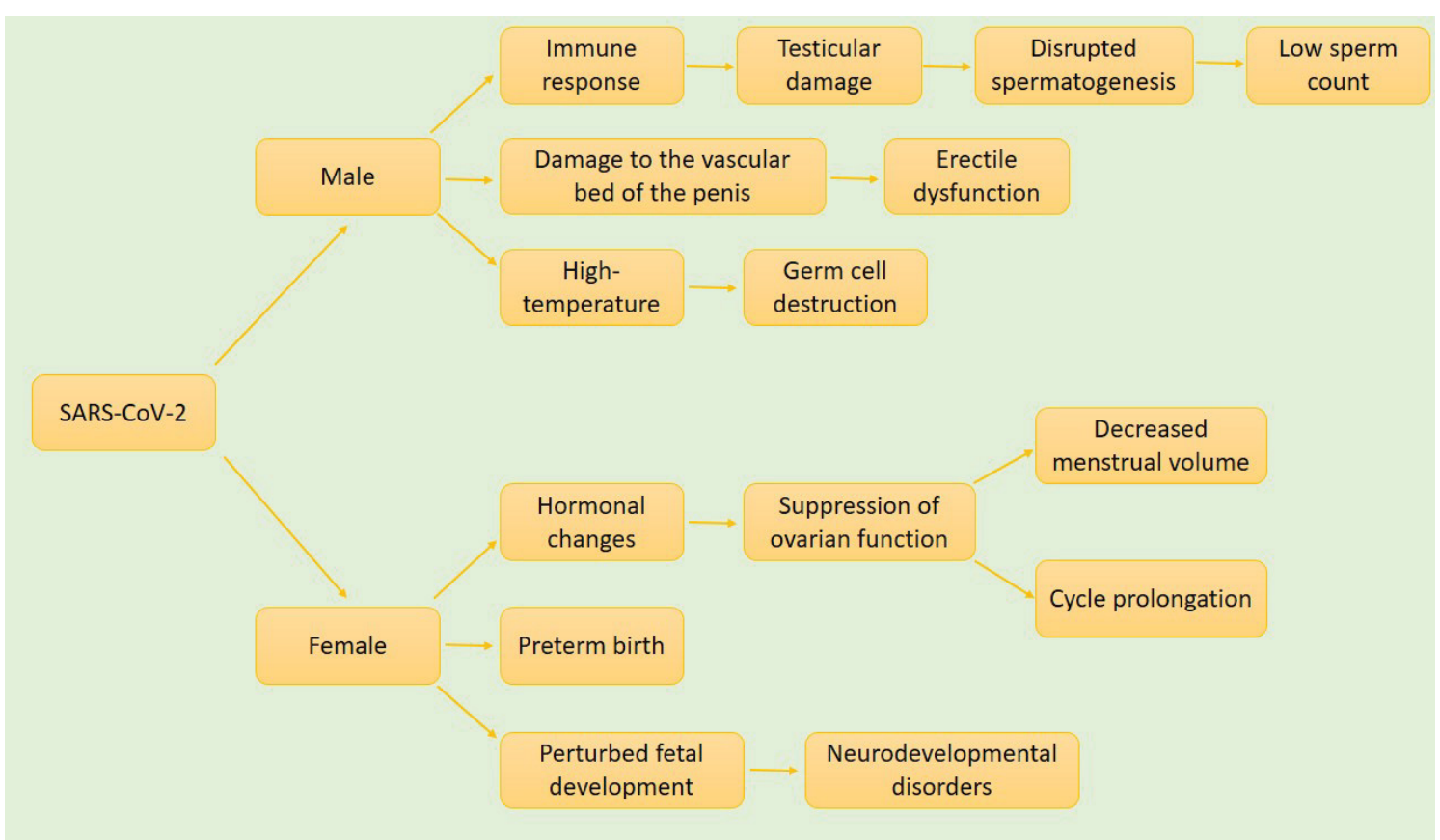

Figure 9: Effect of COVID-19 on the reproductive system. SARS-CoV-2 affects both the male and female reproductive systems. In males, exaggerated immune response leads to testicular damage, disrupted spermatogenesis, and consequently low sperm count. SARS-CoV-2 induced damage to the vascular bed of the penis causes erectile dysfunction. This is because the integrity of the vascular bed of the penis is necessary for the erectile function of the penis. Inflammation-induced high temperature destroys germ cells as well. In females, hormonal changes suppress ovarian function, leading to decreased menstrual volume and cycle prolongation. In pregnant women, it contributes to preterm birth and perturbed fetal development, which can result in a wide range of neurodevelopmental disorders.

caused by suppression of ovarian function that returns to its normal function after recovery [55] (Figure 9).

\section{Potential Therapeutic Approaches}

Several efforts have been made and are still in progress to produce a vaccine and drugs to tackle COVID-19. Convalescent plasma (CP) therapy has been proposed to be a promising cure for COVID-19. In this therapy, plasma containing neutralizing antibodies is taken from a donor who has fully recovered from SARSCoV-2 infection and then administered to infected patients. Soluble human ACE2 has also been proposed to be a therapy for COVID-19. It was suggested that hindering the interaction between SARS-CoV-2 and ACE2 receptors could be used as an effective treatment in COVID-19. Several other anti-retroviral drugs have been could also be promising therapy for COVID-19 [56,57]. According to Grimes, et al., Arginine depletion may also be an effective therapeutic approach against COVID-19. Arginine is an amino acid that is important in the life cycle of many viruses. Therefore, depleting this arginine can interrupt the progression of COVID-19 [58]. Antiviral drugs as a therapeutic approach are exploring two methods in general; first, they prevent the entry of the virus into the host's cells, and secondly, they suppress various steps in the replication process of the virus inside the host's cells [59]. Since inflammation is one of the major factors responsible for disease progression in COVID-19, it was proposed that anti-inflammatory therapies could improve patient symptoms and when properly used and maintained could be a promising therapy for COVID-19 [60]. In addition, the therapeutic probability of estrogen and oxytocin against COVID-19 remains a subject of extensive research. Several research efforts are still ongoing on therapeutic approaches to tackle COVID-19.

\section{Conclusion}

In conclusion, SARS-CoV-2 has been demonstrated to be capable of affecting multiple organ systems of the body. Although the majority of the clinical manifestations of the disease are related to the respiratory system because it is the route of entry and the major target of the disease. However, many of its clinical symptoms are also manifested in the other systems of the body such as the nervous system, cardiovascular system, etc. Some mechanisms behind these effects have been established and the most common mechanism is exaggerated immune and inflammatory response which is the leading cause of tissue and organ damage in COVID-19. Several studies are explaining the mechanism of damage caused by SARS-CoV-2 on the respiratory system, but the mechanisms of these effects on many other systems, such as the cardiovascular system are still unclear. It is hereby highly recommended that further research should be done to elucidate the pathophysiology of this disease, which could give a hint to developing an effective therapy against the disease.

\section{Author Contributions}

Authors contributed equally to the review manuscript. 


\section{Conflict of Interest}

Authors declare no conflict of interest.

\section{References}

1. Stasi C, Fallani S, Voller F, Silvestri C (2020) Treatment for COVID-19: An overview. Eur J Pharmacol 889: 173644.

2. Aluko OM, Lawal SA, Falana MM, Adeagbo AS, ljomone OM (2021) Tackling COVID-19 in Africa: A focus on Nigeria's peculiarities and challenges. Innovation 2: 100078.

3. Shi Y, Wang G, Cai X-P, Deng J-W, Zheng L, et al. (2020) An overview of COVID-19. J Zhejiang Univ Sci B 21: 343360.

4. Gao Z, Xu Y, Sun C, Wang X, Guo Y, et al. (2021) A systematic review of asymptomatic infections with COVID-19. J Microbiol Immunol Infect 54: 12-16.

5. Wang M, Liao Z (2020) SARS-CoV-2 and COVID-19: How much do we know? Acta Virol 64: 288-296.

6. Ejazi SA, Ghosh S, Ali N (2021) Antibody detection assays for COVID-19 diagnosis: An early overview. Immunol Cell Biol 99: 21-33.

7. Umakanthan S, Sahu P, Ranade AV, Bukelo MM, Sushil Rao J, et al. (2020) Origin, transmission, diagnosis and management of coronavirus disease 2019 (COVID-19). Postgrad Med J 96: 753-758.

8. Gul R, Kim UH, Alfadda AA (2021) Renin-angiotensin system at the interface of COVID-19 infection. Eur J Pharmacol 890: 173656.

9. Wiersinga WJ, Rhodes A, Cheng AC, Peacock SJ, Prescott HC (2020) Pathophysiology, transmission, diagnosis, and treatment of coronavirus disease 2019 (COVID-19): A Review. JAMA 324: 782-793.

10. Kumar M, Al Khodor S (2020) Pathophysiology and treatment strategies for COVID-19. J Transl Med 18: 353.

11. Xu Z, Shi L, Wang Y, Zhang J, Huang L, et al. (2020) Pathological findings of COVID-19 associated with acute respiratory distress syndrome. Lancet Respir Med 8: 420422.

12. South AM, Diz DI, Chappell MC (2020) COVID-19, ACE2, and the cardiovascular consequences. Am J Physiol Heart Circ Physiol 318: H1084-H1090.

13. Zheng Y-Y, Ma Y-T, Zhang J-Y, Xie X (2020) COVID-19 and the cardiovascular system. Nat Rev Cardiol 17: 259260.

14. Liu PP, Blet A, Smyth D, Li H (2020) The science underlying COVID-19: Implications for the cardiovascular system. Circulation 142: 68-78.

15. Abboud H, Abboud FZ, Kharbouch H, Arkha Y, El Abbadi $\mathrm{N}$, et al. (2020) COVID-19 and SARS-Cov-2 Infection: Pathophysiology and clinical effects on the nervous system. World Neurosurg 140: 49-53.

16. ladecola C, Anrather J, Kamel H (2020) Effects of COVID-19 on the nervous system. Cell 183: 16-27.e1.

17. Iroegbu JD, Ifenatuoha CW, ljomone OM (2020) Potential neurological impact of coronaviruses: Implications for the novel SARS-CoV-2. Neurol Sci 41: 1329-1337.

18. Heneka MT, Golenbock D, Latz E, Morgan D, Brown $R$ (2020) Immediate and long-term consequences of COVID-19 infections for the development of neurological disease. Alzheimers Res Ther 12: 69.
19. Walling AD, Dickson G (2013) Guillain-Barré syndrome. Am Fam Physician 87: 191-197.

20. Whittaker A, Anson M, Harky A (2020) Neurological manifestations of COVID-19: A systematic review and current update. Acta Neurol Scand 142: 14-22.

21. Armaly Z, KinanehS, SkoreckiK (2021)Renal manifestations of Covid-19: Physiology and pathophysiology. J Clin Med 10: 1216

22. Shao M, Li X, Liu F, Tian T, Luo J, et al. (2020) Acute kidney injury is associated with severe infection and fatality in patients with COVID-19: A systematic review and metaanalysis of 40 studies and 24,527 patients. Pharmacol Res 161: 105107.

23. Yang X, Jin Y, Li R, Zhang Z, Sun R, et al. (2020) Prevalence and impact of acute renal impairment on COVID-19: A systematic review and meta-analysis. Crit Care 24: 356.

24. Diao B, Wang C, Wang R, Feng Z, Zhang J, et al. (2021) Human kidney is a target for novel severe acute respiratory syndrome coronavirus 2 infection. Nat Commun 12: 2506.

25. Martinez-Rojas MA, Vega-Vega O, Bobadilla NA (2020) Is the kidney a target of SARS-CoV-2? Am J Physiol Renal Physiol 318: F1454-f1462.

26. Alshebri MS, Alshouimi RA, Alhumidi HA, Alshaya Al (2020) Neurological complications of SARS-CoV, MERS-CoV, and COVID-19. SN Compr Clin Med 2: 2037-2047.

27. Katyal N, Naural N, Acharya S, Govindarajan R (2020) Neuromuscular complications with SARS-COV-2 infection: A review. Front Neurol 11: 1052.

28. Galassi G, Marchioni A (2020) Facing acute neuromuscular diseases during COVID-19 pandemic: Focus on GuillainBarré syndrome. Acta Neurol Belg 120: 1067-1075.

29. Hasset CE, Gedansky A, Migdady I, Bhimraj A, Uchino K, et al. (2020) Neurologic complications of COVID-19. Cleve Clin J Med 87: 729-734.

30. Henry BM (2020) COVID-19, ECMO, and lymphopenia: A word of caution. Lancet Respir Med 8: e24.

31. Karimi SM, Niazkar HR, Rad F (2021) COVID-19 and hematology findings based on the current evidences: $A$ puzzle with many missing pieces. Int J Lab Hematol 43: 160-168.

32. Zhang JJ, Dong X, Cao Y-Y, Yuan Y-D, Yang Y-B, et al. (2020) Clinical characteristics of 140 patients infected with SARS-CoV-2 in Wuhan, China. Allergy 75: 1730-1741.

33. Zhao Q, Meng M, Kumar R, Wu Y, Huang J, et al. (2020) Lymphopenia is associated with severe coronavirus disease 2019 (COVID-19) infections: A systemic review and meta-analysis. Int J Infect Dis 96: 131-135.

34. Fan BE, Chong VCL, Chan SSW, Lim GH, Lim KGE, et al. (2020) Hematologic parameters in patients with COVID-19 infection. Am J Hematol 95: E131-E134.

35. Giamarellos-Bourboulis EJ, Netea MG, Rovina N, Akinosoglou K, Antoniadou A, et al. (2020) Complex immune dysregulation in COVID-19 patients with severe respiratory failure. Cell Host Microbe 27: 992-1000.e3.

36. Liu F, Xu A, Zhang Y, Xuan W, Yan T, et al. (2020) Patients of COVID-19 may benefit from sustained Lopinavircombined regimen and the increase of Eosinophil may predict the outcome of COVID-19 progression. Int $\mathrm{J}$ Infect Dis 95: 183-191.

37. Paces J, Strizova Z, Smrz D, Cerny J (2020) COVID-19 and the immune system. Physiol Res 69: 379-388. 
38. Almeida JFM, Chehter EZ (2020) COVID-19 and the gastrointestinal tract: What do we already know? Einstein (Sao Paulo) 18: eRW5909.

39. Galanopoulos M, Gkeros F, Doukatas A, Karianakis G, Pontas C, etal.(2020)COVID-19 pandemic:Pathophysiology and manifestations from the gastrointestinal tract. World $\mathrm{J}$ Gastroenterol 26: 4579-4588.

40. Wong SH, Lui RN, Sung JJ (2020) Covid-19 and the digestive system. J Gastroenterol Hepatol 35: 744-748.

41. Wastnedge EAN, Reynolds RM, van Boeckel SR, Stock SJ, Denison FC, et al. (2021) Pregnancy and COVID-19. Physiol Rev 101: 303-318.

42. Jothimani D, Venugopal R, Abedin MF, Kaliamoorthy I, Rela M (2020) COVID-19 and the liver. J Hepatol 73: 12311240.

43. Patel KP, Patel PA, Vunnam RR, Hewlett AT, Jain R, et al. (2020) Gastrointestinal, hepatobiliary, and pancreatic manifestations of COVID-19. J Clin Virol 128: 104386.

44. Cha MH, Regueiro M, Sandhu DS (2020) Gastrointestina and hepatic manifestations of COVID-19: A comprehensive review. World J Gastroenterol 26: 2323-2332.

45. Lundholm MD, Poku C, Emanuele N, Emanuele MA, Lopez N (2020) SARS-CoV-2 (COVID-19) and the endocrine system. J Endocr Soc 4: bvaa144.

46. Pal R, Banerjee M (2020) COVID-19 and the endocrine system: Exploring the unexplored. J Endocrinol Invest 43: 1027-1031.

47. Marazuela M, Giustina A, Puig-Domingo M (2020) Endocrine and metabolic aspects of the COVID-19 pandemic. Rev Endocr Metab Disord 21: 495-507.

48. Anderson G, Reiter RJ (2020) Melatonin: Roles in influenza, Covid-19, and other viral infections. Rev Med Virol 30: e2109.

49. Meng TT, Dong RJ, Li TG (2021) Relationship between COVID-19 and the male reproductive system. Eur Rev Med Pharmacol Sci 25: 1109-1113.
50. Groner MF, de Carvalho RC, Camillo J, Ferreira PRA, Fraietta $R$ (2021) Effects of Covid-19 on male reproductive system. Int Braz J Urol 47: 185-190.

51. Sansone A, Mollaioli D, Ciocca G, Limoncin E, Colonnello $\mathrm{E}$, et al. (2021) Addressing male sexual and reproductive health in the wake of COVID-19 outbreak. J Endocrinol Invest 44: 223-231.

52. Sheikhzadeh HF, Hosseinzadeh SS, Asl Monadi Sardroud MA (2021) Review of COVID-19 and male genital tract. Andrologia 53: e13914.

53. Jing $Y$, Run-Qian L, Hao-Ran W, Hao-Ran C, Ya-Bin L, et al. (2020) Potential influence of COVID-19/ACE2 on the female reproductive system. Mol Hum Reprod 26: 367-373.

54. Moore KM, Suthar MS (2021) Comprehensive analysis of COVID-19 during pregnancy. Biochem Biophys Res Commun 538: 180-186.

55. Li K, Chen G, Hou H, Liao Q, Chen J, et al. (2021) Analysis of sex hormones and menstruation in COVID-19 women of child-bearing age. Reprod Biomed Online 42: 260-267.

56. Iyer M, Jayaramayya K, Subramaniam MD, Lee SB, Abdal Dayem A, et al. (2020) COVID-19: An update on diagnostic and therapeutic approaches. BMB Rep 53: 191-205.

57. Uddin M, Mustafa F, Rizvi TA, Loney T, Al Suwaidi $H$, et al. (2020) SARS-CoV-2/COVID-19: Viral genomics, epidemiology, vaccines, and therapeutic interventions. Viruses 12: 526.

58. Grimes JM, Khan S, Badeaux M, Rao RM, Rowlinson SW, et al. (2021) Arginine depletion as a therapeutic approach for patients with COVID-19. Int J Infect Dis 102: 566-570.

59. Majumder J, Minko T (2021) Recent developments on therapeutic and diagnostic approaches for COVID-19. AAPS J 23: 14.

60. lannaccone G, Scacciavillani R, Del Buono MG, Camilli M, Ronco C, et al. (2020) Weathering the Cytokine Storm in COVID-19: Therapeutic implications. Cardiorenal Med 10: 277-287. 